\title{
Osteoporosis: impact on health and economics
}

Nicholas Harvey, Elaine Dennison and Cyrus Cooper

\begin{abstract}
Osteoporosis is a major public health problem through associated fragility fractures. The most common sites of fracture are the hip, spine and wrist, and these have an enormous health and economic impact. All fractures result in some degree of morbidity, but fractures at the hip are associated with the worst outcomes. The worldwide direct and indirect annual costs of hip fracture in 1990 were estimated at US $\$ 34.8$ billion, and are expected to increase substantially over the next 50 years. Fracture incidence varies between populations, and is set to increase over coming decades as the global population becomes more elderly. This effect will be particularly marked in the developing world, which is additionally assuming morewesternized lifestyles that predispose to increased fracture risk. Strategies to target those at high risk of fracture have been developed, but preventative measures at the public health level are also urgently needed to reduce the burden of this devastating disease.
\end{abstract}

Harvey, N. et al. Nat. Rev. Rheumatol. 6, 99-105 (2010); doi:10.1038/nrrheum.2009.260

\section{Introduction}

Osteoporosis is a skeletal disease characterized by low bone mass and microarchitectural deterioration of bone tissue, which results in increased bone fragility and susceptibility to fracture. ${ }^{1}$ Historically, osteoporosis has been difficult to define in clinical terms: a focus on bone mineral density (BMD) does not encompass all the risk factors for fracture, whereas a fracture-based definition precludes identification of at-risk populations. In 1994, the WHO convened to resolve this issue, subsequently defining osteoporosis in terms of both BMD and previous fracture. Therefore, osteoporosis is diagnosed if the BMD measured by dual X-ray absorptiometry is more than 2.5 standard deviations below the sex-matched young adult mean; if this criterion is met in a patient with a history of fragility fractures, the term 'established osteoporosis' is used. ${ }^{2}$

Osteoporosis is a highly prevalent disease and results in massive costs both to the individual and to society through associated fragility fractures. The most common sites of fragility fractures are the hip, spine and distal forearm, and the impact of such fractures manifests in terms of mortality, morbidity and economic cost. This Review will explore the consequences of fractures in the context of the overall epidemiology of osteoporosis.

\section{Fracture epidemiology}

\section{Incidence and prevalence}

The 2004 US Surgeon General's report highlighted the enormous burden of osteoporosis-related fracture. ${ }^{3}$ An estimated 10 million Americans over the age of 50 years have osteoporosis, and around 1.5 million fragility fractures occur in these patients each year. An additional 34 million Americans are at risk of the disease. The

Competing interests

The authors declare no competing interests. report estimates that the risk of fragility fracture for a 50 year old women in her remaining lifetime could approach one in two; the figure for men is one in five. According to van Staa et al. ${ }^{4}$ the risk of fragility fractures seems to be similar in the UK. ${ }^{4}$ Fracture incidence in the community shows peaks in youth and in the very elderly. Fractures of long bones, usually after substantial trauma, predominate in children and young adults and are more frequent in males than females. In addition, data suggest that reduced bone strength could be of relevance to the fracture risk in young individuals. ${ }^{5}$

\section{Hip fracture}

The incidence of hip fracture in most populations increases exponentially with age (Figure 1). Above 50 years of age, there is a male:female incidence ratio of around 1:2. ${ }^{6}$ Overall, approximately $98 \%$ of hip fractures occur among people aged 35 years and over, and $80 \%$ occur in women (because there are more elderly women than men). In 1990, an estimated 1.66 million hip fractures occurred worldwide, of which 1.19 million were in women and 463,000 in men. ${ }^{7}$ Most hip fractures occur after a fall from standing height or less, ${ }^{8}$ and the direction of the fall is known to be important: a sideways fall directly onto the hip is more likely to cause a fracture than is falling forwards. ${ }^{9}$ An analysis of the General Practice Research Database (GPRD, which includes $6 \%$ of the UK population) in 1993 revealed that the lifetime risk of hip fracture for 50 year olds in the UK is $11.4 \%$ and $3.1 \%$ for women and men, respectively. ${ }^{4}$ Most of the increased risk is gained in old age, such that a 50 year old woman's 10 -year risk of hip fracture is $0.3 \%$, increasing to $8.7 \%$ at 80 years of age. ${ }^{4}$ The corresponding figures for men are $0.2 \%$ and $2.9 \%$ at 50 and 80 years of age, respectively.

Hip fracture incidence varies by season, with an increase during winter in temperate countries. ${ }^{10}$ Their
The MRC Epidemiology Resource Centre, University of Southampton, Southampton General Hospital, Southampton S016 6YD, UK

(N. Harvey, E. Dennison, C. Cooper).

Correspondence to: C. Cooper cc@mrc.soton.ac.uk 


\section{REVIEWS}

\section{Key points}

- Osteoporosis-related fractures are very common, and are associated with high direct and indirect costs to the global economy

- Hip and vertebral fractures are associated with impaired quality of life and a $20 \%$ reduction in survival

- The number of fractures will increase globally with the aging population, with much of the future burden falling on the developing world

- New case-finding strategies, such as FRAX ${ }^{\circledR}$, offer the potential to improve the targeting of individuals most at risk

- Studies in developed populations have suggested modest reductions in the age-adjusted and gender-adjusted rates of osteoporosis-associated fracture
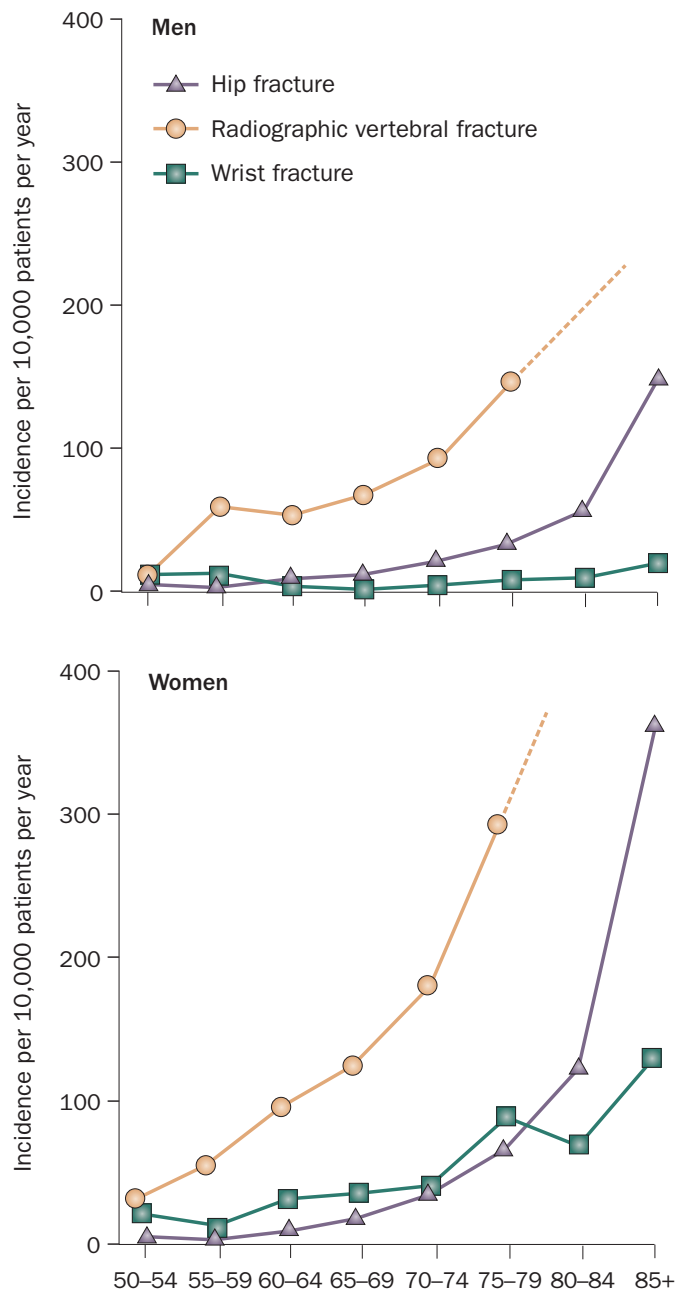

Age group (years)

Figure 1 | The incidence of radiographically determined vertebral, hip and wrist fracture by age and gender. ${ }^{4,14}$ Reprinted with permission from Elsevier from Sambrook, P. \& Cooper, C. Osteoporosis. Lancet 367, 2010-2118 (2006).

mainly indoor occurrence would imply that this increase is not a result of slipping on icy pavements; rather, possible causes include slowed neuromuscular reflexes and lower ambient light in winter weather. The latter is likely to lead to reduced levels of circulating 25-hydroxyvitamin D, with a consequent adverse effect on bone metabolism and muscle function.

\section{Vertebral fracture}

Studies of vertebral fracture prevalence are probably best viewed as demonstrating the frequency of 'vertebral deformity', as many vertebrae will be of abnormal shape for reasons other than fracture, such as in Scheuermann disease (also known as Scheuermann kyphosis or osteochondrosis of the spine) and developmental variations. The term 'fracture' in this context is best reserved for the occurrence of incident shape change. Data from the European Vertebral Osteoporosis Study (EVOS) demonstrated that the age-standardized population prevalence of vertebral deformity across Europe was $12.2 \%$ for men and $12.0 \%$ for women aged $50-79$ years. ${ }^{11}$ In the past it was thought that vertebral deformities were more common in women than men, but the EVOS data suggest that this is not the case at younger ages. According to this study, the prevalence of deformities in 50-60 year olds is similar between the sexes, if not higher in men, possibly as a result of a greater incidence of trauma in this group. ${ }^{11}$ Additionally, work from Australia, using the Dubbo Osteoporosis Epidemiology Study cohort, has demonstrated a higher prevalence of vertebral deformity in men than women at older ages. ${ }^{12}$

Differences in detection threshold and definition of deformity or fracture might play a part in this variation. Most vertebral fractures in elderly women occur as a result of everyday activities such as lifting, rather than through falling. Many vertebral fractures are asymptomatic, and there is disagreement about the radiographic definition of such fractures. As a result, in population studies that use radiographic screening, the incidence of all vertebral fractures has been estimated to be three times that of hip fracture, with only one-third of these coming to medical attention. ${ }^{13}$ Data from EVOS have enabled accurate assessment of radiographically determined vertebral fracture in a large population. At age 75-79 years, the incidence of vertebral fractures so-defined was 13.6 per 1,000 person-years for men and 29.3 per 1,000 personyears for women. ${ }^{14}$ This compares with 0.2 per 1,000 person-years for men and 9.8 per 1,000 person-years in 75-84 year olds where fractures were defined by clinical presentation (but confirmed by radiograph) in an earlier study from Rochester, MN in the USA. ${ }^{13}$ The overall age-standardized incidence of vertebral fracture in the European Prospective Osteoporosis Study (EPOS) was 10.7 per 1,000 person-years in women and 5.7 per 1,000 person-years in men. ${ }^{14}$

\section{Wrist fracture}

Wrist fractures show a pattern of occurrence that differs from that of hip and vertebral deformities. The incidence of this type of fracture increases in white women from the age of 45 to 60 years, followed by a plateau. ${ }^{10}$ This plateau might relate to altered neuromuscular reflexes seen with aging, which are associated with a tendency to fall sideways or backwards, meaning that the fall is not stopped with an outstretched arm. Most wrist fractures occur in 
women, and $50 \%$ occur in women aged over 65 years. Data from the UK GPRD have shown that the lifetime risk of wrist fracture in a 50 year old woman is $16.6 \%$, falling to $10.4 \%$ by 70 years of age. ${ }^{4}$ By contrast, the incidence in men is low (lifetime risk of $2.9 \%$ at age 50 years and $1.4 \%$ at age 70 years). ${ }^{4}$

\section{Geographic variation in incidence and prevalence}

The incidence of hip fracture varies within populations of a given race or sex. Age-adjusted hip fracture incidence rates are higher among white residents of Scandinavia than comparable patients in the USA or Oceania. ${ }^{15-17}$ Among European countries, the range of variation is approximately 11 -fold. ${ }^{16}$ These variations cannot be explained by differences in activity levels, alcohol consumption, smoking, obesity or migration status. ${ }^{15}$ The EVOS study demonstrated that patients from Scandinavia had the highest rates of vertebral deformity, with a threefold difference in the prevalence between countries across Europe. ${ }^{11}$ The range among European centers was 7.5$19.8 \%$ for men and $6.2-20.7 \%$ for women. The differences in the incidence of vertebral deformity are not as great as those seen for hip fracture in Europe, however, and some of these differences can be explained by variations in physical activity and BMI. ${ }^{11}$

\section{Clustering of fractures in individuals}

The presence of a prevalent vertebral deformity leads to a 7-10-fold increase in the risk of subsequent vertebral deformities. ${ }^{18}$ This increase in risk is similar to that of patients who have sustained one hip fracture to then experience another. In addition, data from the Rochester cohort suggest that a distal forearm fracture is associated with 1.4-fold increase in the risk of subsequent hip fracture in women and a 2.7 -fold increase in men. ${ }^{19}$ The corresponding values for subsequent vertebral fracture are 5.2 and 10.7 in women and men, respectively. Data from EPOS demonstrate that prevalent vertebral deformity is a strong predictor of incident hip fracture (with a rate ratio of 2.8-4.5), and the risk of hip fracture increases with the number of previous vertebral deformities. ${ }^{20}$ The number and morphometry of baseline vertebral deformities also predict the occurrence of incident vertebral fracture. ${ }^{21}$ The incidence of new vertebral fracture within 1 year of an incident vertebral fracture is $19.2 \%,{ }^{22}$ and in the Rochester cohort the cumulative incidence of any fracture 10 years after the baseline event was $70 \%{ }^{19}$

These findings emphasize the importance of prompt therapeutic action on discovery of vertebral deformities. This notion is further supported by a recent study from the Netherlands, in which 4,140 postmenopausal women were followed for 5 years. ${ }^{23}$ In this cohort, 23\% had refractured within 1 year of an initial fracture, and $54 \%$ had refractured within 5 years. The relative risk of subsequent fracture declined with time from the initial fracture: thus, the relative risk of subsequent fracture in the first year was 5.3 , which decreased to 2.8 within 2-5 years, 1.4 within 6-10 years, and dropped to 0.41 at more than 10 years after the initial fracture. Finally, data from the Dubbo Osteoporosis Epidemiology Study

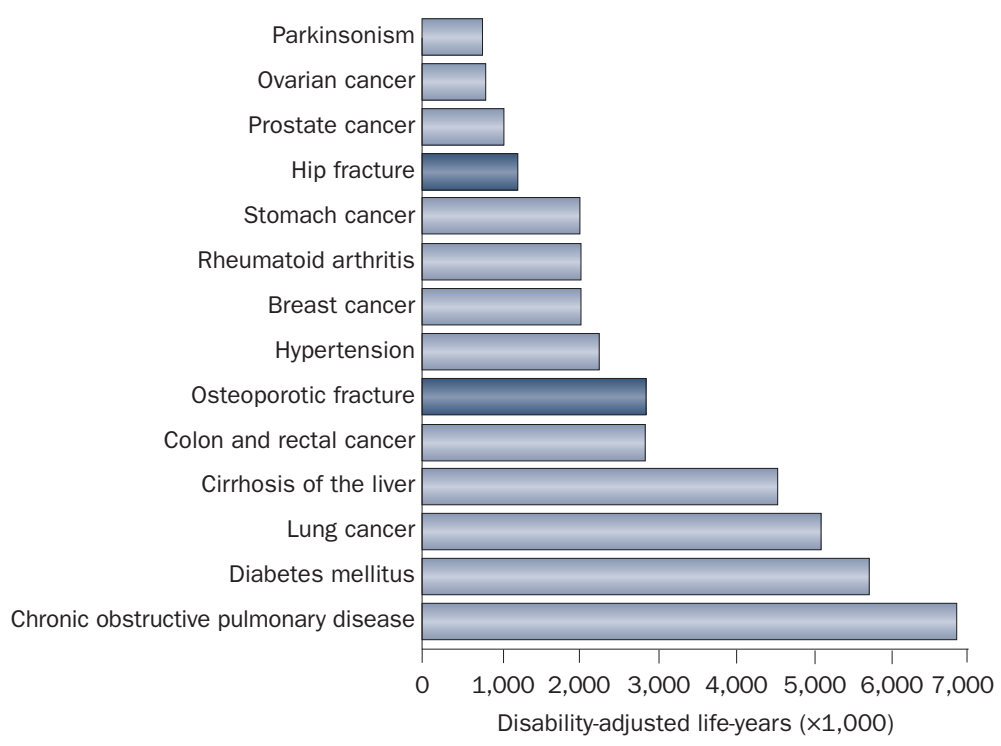

Figure 2 | The burden of diseases estimated as disability-adjusted life-years in 2002 in the Americas and Europe combined. ${ }^{26}$ Reproduced with kind permission from Springer Science \& Business Media: Johnell, O. \& Kanis, J. A. An estimate of the worldwide prevalence and disability associated with osteoporotic fracture. Osteoporos. Int. 17, 1726-1733 (2006).

suggested that refracture rates in men and women were similar over a 10-year period, and that most types of fracture were associated with an increased risk of subsequent fracture, apart from those in the ribs in men or ankles in women. ${ }^{24}$

\section{Health impact of fragility fracture}

Fragility fractures are associated with significant morbidity; both hip fractures and vertebral deformities are additionally associated with excess mortality. An estimated 740,000 deaths per year are associated with hip fracture. ${ }^{25}$ Fragility fractures account for $0.83 \%$ of the burden of noncommunicable disease worldwide and $1.75 \%$ in Europe, where they are associated with more disability-adjusted life years than many other chronic noncommunicable diseases (Figure 2). ${ }^{26}$

\section{Mortality}

Data from the Rochester cohort showed that survival rates 5 years after hip and vertebral fractures were approximately $80 \%$ of those expected for men and women of similar age without fractures (Figure 3) ${ }^{27}$ Mortality associated with hip fracture increases with age, is higher in men than women, and is greater for those with poor prefracture functional status and coexisting illnesses. Around 31,000 excess deaths occur within 6 months of approximately 300,000 hip fractures every year in the US. ${ }^{28}$ About $8 \%$ of men and $3 \%$ of women aged over 50 years die while in hospital following their hip fracture. ${ }^{28}$ Mortality rates after hip fracture continue to rise over the subsequent months and peak at 1 year, with a rate of $36 \%$ for men (higher for the very elderly) and $21 \%$ for women. ${ }^{28}$ Similarly, in the UK, the 12 -month survival rate post-hip-fracture for men is $63.3 \%$ versus $90.0 \%$ expected, and for women is $74.9 \%$ versus $91.1 \%$ expected. ${ }^{4}$ The 


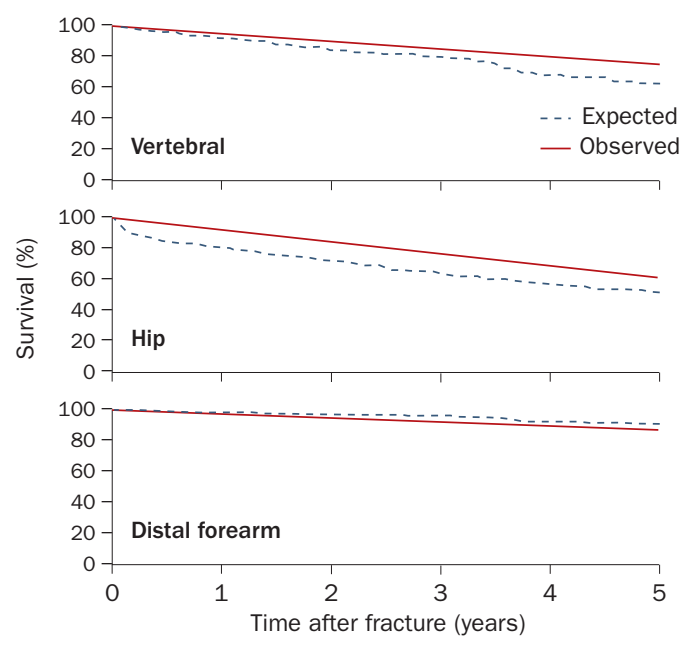

Figure 3 | Survival after osteoporotic fractures. ${ }^{27}$ Reproduced by permission of Oxford University Press from Cooper, C. et al. Population-based study of survival after osteoporotic fractures. Am. J. Epidemiol. 137, 1001-1005 (1993).

risk of death in the UK is maximal immediately after the fracture and decreases gradually over time. However, data from the Dubbo Epidemiology Study of communitydwelling women and men aged at least 60 years suggest that elevated mortality persists for up to 10 years after hip fracture. ${ }^{29} \mathrm{~A}$ subsequent fracture further elevates the risk of death over another 5 years. In this cohort, survival was reduced following all types of fracture except for minor fractures, where mortality was increased only for those aged 75 years or older. Data from Sweden suggest that only $25 \%$ of deaths from hip fracture are directly attributable to the fracture or to complications such as infection, thromboembolism and subsequent surgery. ${ }^{30}$ The residual mortality might reflect coexisting morbidity from underlying diseases, although, in the Dubbo study, the main predictors of higher mortality were male sex, increasing age, quadriceps weakness and subsequent fracture, but not the presence of comorbidities. Smoking, low BMD and body sway (also known as postural sway-slight movements related to maintenance of balance) were also predictors of post-fracture mortality in women, as was a lower level of physical activity in men. ${ }^{29}$

The pattern and causes of death associated with hip fracture might differ somewhat for those in institutional care. A recent Australian nested case-control study of 2005 elderly people in residential care found that survival rates for hip fracture and control patients were similar within 1 year following fracture repair. ${ }^{31}$ After adjusting for sex, gender, institution type, weight, immobility, cognitive function, comorbidities and number of medications, the hazard ratio of death following surgery for hip fracture for patients compared with controls was 3.09 after the first 3 months, 1.99 at 3-9 months and 0.88 at more than 9 months post-fracture repair. The main causes of death in the first 9 months were infections for females and cardiac disease for both sexes. Interestingly, bisphosphonate use was associated with a reduction in mortality post-fracture repair. ${ }^{31}$ It seems likely that the swift equalization of mortality rates between cases and controls in this population reflects the high risk of death in institutionalized populations compared with free-living individuals in the GPRD and Dubbo studies.

Given the obesity epidemic faced by much of the developed world, concern has been raised regarding a possible association between obesity and adverse outcomes following surgery for hip fracture. However, this seems not to be the case, at least with regards to noncardiac complications, with recent US data showing a higher risk of adverse outcomes in patients with below-normal BMI, rather than those with above-normal BMI. ${ }^{32}$ Dementia, in addition to age, was found to be an independent predictor of poor outcome following surgery for hip fracture in an Israeli discharge study. ${ }^{33}$ Finally, there is some suggestion of a decrease in mortality following hip fracture, despite increasing rates of comorbidity, in longitudinal comparative data using the Medicare database in the USA. ${ }^{34}$

Excess mortality after vertebral fracture seems to persist for up to 5 years in both sexes. ${ }^{27,29}$ Only $8 \%$ of deaths following vertebral fractures are directly attributable to osteoporosis, and impaired survival is more pronounced for vertebral fractures that follow moderate rather than severe trauma. ${ }^{27}$ In the UK GPRD study, the observed survival in women 12 months after vertebral fracture was $86.5 \%$ versus $93.6 \%$ expected; at 5 years, survival decreased to $56.5 \%$ observed compared with $69.9 \%$ expected. In the Dubbo study the age-adjusted standardized mortality ratio following vertebral fracture was 1.82 for women and 2.12 for men. ${ }^{29}$

Fractures of the radius or ulna tend not to be associated with increased mortality except in elderly men, where only a slight excess of deaths has been observed. ${ }^{27}$

\section{Morbidity}

Of those who survive a fragility fracture, many will remain significantly impaired. In the USA, $7 \%$ of survivors of all types of fracture have some degree of permanent disability, and $8 \%$ require long-term institutional care. ${ }^{35}$ Overall, a 50 year old white American woman has a $13 \%$ chance of experiencing functional decline after any fracture. ${ }^{35}$ Table 1 summarizes the impact of hip, spine and wrist fractures on morbidity.

Consistent with their effect on mortality, hip fractures contribute most to osteoporosis-associated disability. Patients are prone to developing acute complications such as pressure sores, bronchopneumonia and urinary tract infections. Long-term mobility may be severely impaired; $50 \%$ of those who are ambulatory before the fracture are unable to walk independently afterwards. Age is an important predictor of outcome, with $14 \%$ of 50-55 year old hip fracture patients being discharged to residential care homes compared with $55 \%$ of those aged over 90 years. ${ }^{35}$ In the USA, $25 \%$ of formerly independent patients became at least partially dependent following a hip fracture, and $50 \%$ of those who were dependent prefracture were admitted to residential care; those who were already in care homes remained there. ${ }^{35}$ 
Although only a minority of vertebral fractures come to clinical attention, symptomatic vertebral fractures account for 52,000 hospital admissions in the USA and 2,188 in England and Wales each year in patients aged 45 years and over. ${ }^{36,37}$ The major clinical consequences of vertebral fracture are back pain, kyphosis, and height loss. Scores on the quality of life questionnaire of the European Foundation for Osteoporosis decrease as the number of vertebral fractures increases. ${ }^{38}$ The psychological impact of functional loss can lead to depression and social isolation, as well as to a loss of self-esteem. In EPOS, participants with radiologically identified vertebral fracture at baseline had repeat radiographs performed 3 years later. ${ }^{39}$ Women who had suffered a further fracture during this period experienced substantial levels of disability with impairments in key physical functions of independent living. Few similar data exist for men.

In contrast to fractures of the spine and hip, wrist fractures do not seem to be associated with increased morbidity. ${ }^{4,27}$ Although wrist fractures can impact some activities, such as writing or meal preparation, few patients are completely disabled, despite over half reporting only fair-to-poor function at 6 months post-fracture. ${ }^{35}$

\section{Secular trends and economic cost Future projections}

The economic cost of osteoporosis needs to be understood in terms of both the current and the future burden of fracture. Figure 4 summarizes the secular changes for hip fracture over the last century in Europe, demonstrating the mainly upward trend in age-adjusted incidence to the present time. ${ }^{40}$ The global life expectancy is increasing steadily and the number of elderly individuals is rising in every geographic region. By the year 2050, the population of individuals aged 65 years and over is expected to increase from 323 million to 1,555 million. These demographic changes could lead to an increase in the number of hip fractures occurring worldwide among individuals aged at least 35 years; in fact, the incidence of hip fracture is estimated to increase from 1.66 million in 1990 to 6.26 million in $2050 .{ }^{41}$ Assuming a constant agespecific risk of hip fracture, an increase in the number of over 65 year olds in the USA from 32 million in 1990 to 69 million in 2050 could be accompanied by a threefold rise in the number of hip fractures. ${ }^{41}$ Similarly, in the UK, the number of hip fractures could increase from 46,000 in 1985 to 117,000 in $2016 .{ }^{42}$ By contrast, recent studies from Switzerland and Finland suggest that the age-adjusted incidence of hip fracture has declined over the last decade. ${ }^{43,44}$ The reason for these changes might include an increase in obesity or better screening and treatment for osteoporosis, and could partly offset the impact of the projected increase in the elderly population. This reduction in age-adjusted incidence has not been recorded in the developing world; therefore, the increase in the elderly population together with the adoption of more-westernized lifestyles in these areas is likely to ensure an increase in the worldwide burden of fragility fractures in future generations. This increase is likely to be uneven across the globe, with the expansion of the
Table 1 | Impact of osteoporosis-related fractures in the UK ${ }^{47 * \pm}$

\begin{tabular}{llll}
\hline Impact & Hip & Spine & Wrist \\
\hline $\begin{array}{l}\text { Lifetime risk (\%) } \\
\text { Women }\end{array}$ & 14 & 28 & 13 \\
$\quad$ Men & 3 & 6 & 2 \\
\hline Patients per year & 70,000 & 120,000 & 50,000 \\
Hospitalization (\%) & 100 & $2-10$ & 5 \\
\hline Relative survival & 0.83 & 0.82 & 1.00 \\
\hline *Reproduced with permission from Cooper, C. Epidemiology of osteoporosis. Osteoporos. Int. 9 (Suppl. 2), \\
S2-S8 (1999). Fost for all sites combined: approximately UKf1.7 billion.
\end{tabular}

Annual change in age-adjusted and gender-adjusted hip fracture incidence (\%)

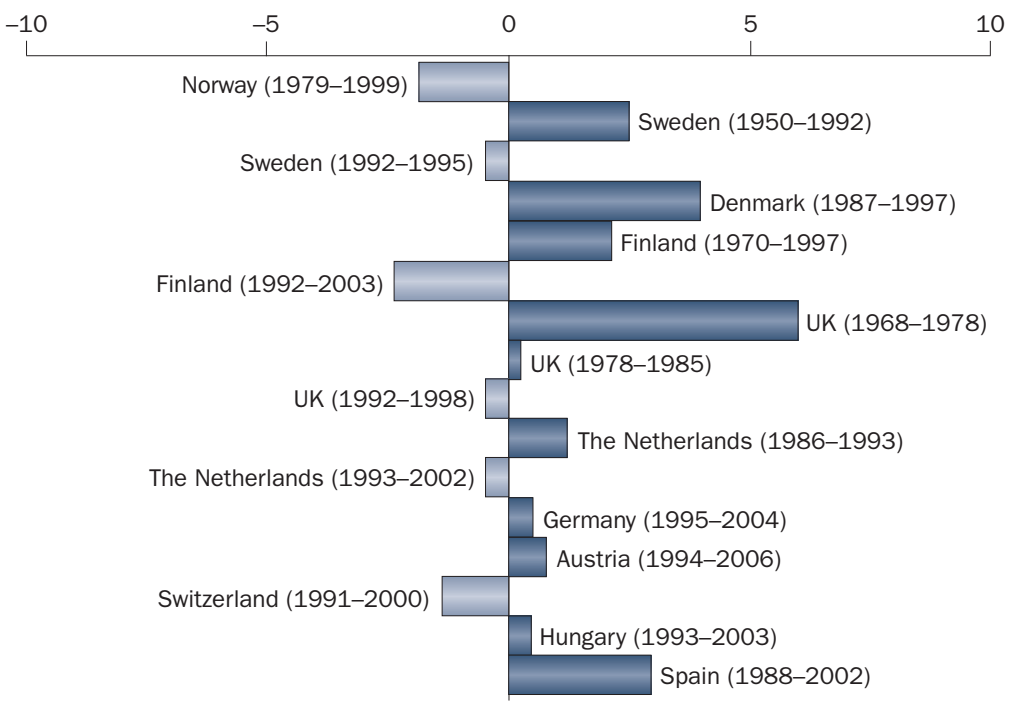

Figure 4 | Secular trends for hip fracture in Europe. ${ }^{40}$

elderly population in Latin America and Asia potentially leading to a shift in the geographical distribution of hip fractures, with only an estimated one-quarter occurring in Europe and North America. ${ }^{41}$

\section{Economic cost and risk assessment strategy}

The cost of osteoporosis-related fractures to the global economy is immense. A conservative estimate of the worldwide direct and indirect annual costs of hip fracture in 1990 was US $\$ 34.8$ billion. This is set to rise to $\$ 131.5$ billion by 2050 (at a cost of $\$ 21,000$ per patient). ${ }^{45}$ The costs associated with other fractures are less welldefined. The cost of fragility fractures for 2005 in the USA was an estimated $\$ 19$ billion. ${ }^{46}$ In the UK alone, the annual cost to the healthcare system from fragility fractures has been estimated at UK£1.7 billion. ${ }^{47}$ Hip fractures account for over one-third of the total UK figure, and reflect the cost of inpatient medical services and residential-home care. Expenditures are rising faster than the general rate of inflation and are a major source of concern to government leaders and third party payers.

\section{Strategies to reduce health and economic impact}

Given the enormous health and economic impact of fragility fracture, there is a clear imperative to develop 
strategies to reduce this burden. Models have been developed to express risk in terms of the 10-year probability of fracture, encompassing hip and other sites. For example, a woman aged 60 years with an average BMD (that is, a T-score of approximately -1.4) might have a 10-year probability of hip fracture of $2.4 \%$; if a previous fragility fracture is included then the risk could increase to $4.8 \%{ }^{48} \mathrm{An}$ example of such a model is the FRAX ${ }^{\circledR}$ algorithm, which was developed using data from nine cohorts around the world. ${ }^{49,50}$ Using this algorithm, clinicians can input easily obtained clinical data, either alone or in combination with $\mathrm{BMD}$, to gain an estimate of the patient's absolute risk of fracture over the next 10 years. This model was recently used in a case-finding strategy for men and women at high risk of fracture, delineating fracture probabilities at which BMD testing or intervention should be recommended. ${ }^{51}$ The results suggested that treatment with generic alendronate therapy (assuming a conservative cost of $£ 95$ per year of treatment) was cost-effective at all ages when the 10-year risk of a major fragility fracture was higher than $7 \%$. The recommended assessment thresholds for BMD testing were $6.9 \%$ at 50 years and $18-36 \%$ at 80 years of age. The thresholds chosen are clearly influenced by a variety of health-economic assumptions, not least the costs of medication and fracture care, as well as the amount society is willing to pay for a given benefit. Further work to validate the $\mathrm{FRAX}^{\circledR}$ algorithm as a case-selection tool in the UK is underway (SCOOP [Screening of Older Women for Prevention of Fracture] Study). Recent data from Australia (dervided from UK and US cohorts) have suggested that $\mathrm{FRAX}^{\circledR}$ is able to discriminate between female patients with fragility fracture and controls, although the results for men were less robust. ${ }^{52}$ Ultimately, similar strategies, in combination with preventative measures, at the public health level could help to reduce the burden of this devastating disease.

\section{Conclusions}

Osteoporosis has a massive impact on health at the individual and population level with a huge monetary cost to national economies. The three most important fragility fractures associated with osteoporosis are those of the hip, spine and wrist. With the global shift towards an aging population, even if age-adjusted incidence of fracture remains stable, there will be a large increase in fracture burden worldwide over the next 50 years, much of which will fall on the developing world. Strategies are being developed to more-comprehensively target those at high risk of fracture both for primary and secondary prevention (such as fracture-liaison services ${ }^{50}$ ), but much more needs to be done at the policy level to reduce the burden of fragility fracture in future generations.

Review criteria
Keywords that were employed to search the PubMed
database included "fracture", "osteoporosis",
"mortality", "survival", "morbidity", "incidence",
"prevalence", "geographic", "economic", "cost",
"clustering", "risk", "secular" and "trends". Articles were
chosen on the basis of relevance, being full-text and in
the English language. No constraints were put on date
of publication. Where appropriate, reference lists were
checked for additional publications.

1. [No authors listed] Consensus development conference: diagnosis, prophylaxis and treatment of osteoporosis. Am. J. Med. 941, 646-650 (1993).

2. WHO. Assessment of fracture risk and its application to screening for postmenopausal osteoporosis. (WHO, Geneva, 1994).

3. US Department of Health and Human Services. Bone health and osteoporosis: a report of the surgeon general (Rockville, MD, USA, 2004).

4. van Staa, T. P., Dennison, E. M., Leufkens, H. G. $\&$ Cooper, C. Epidemiology of fractures in England and Wales. Bone 29, 517-522 (2001).

5. Goulding, A, Jones, I. E., Taylor, R. W., Manning, P. J. \& Williams, S. M. More broken bones: a 4-year double cohort study of young girls with and without distal forearm fractures. J. Bone Miner. Res. 15, 2011-2018 (2000).

6. Melton, L. J. in Osteoporosis: Etiology, Diagnosis and Management (eds Riggs, B. L. \& Melton, L. J.) 133-154 (Raven Press, New York, 1988).

7. Cooper, C. \& Melton, L. J. Epidemiology of osteoporosis. Trends Endocrinol. Metab. 314, 224-229 (1992).

8. Gallagher, J. C., Melton, L. J., Riggs, B. L. \& Bergstrath, E. Epidemiology of fractures of the proximal femur in Rochester, Minnesota. Clin. Orthop. 150, 163-171 (1980).

9. Nevitt, M. C. \& Cummings, S. R. Type of fall and risk of hip and wrist fractures: the study of osteoporotic fractures. The Study of Osteoporotic Fractures Research Group. J. Am. Geriatr. Soc. 41, 1226-1234 (1993).
10. Melton, L. J. \& Cooper, C. in Osteoporosis $2^{\text {nd }}$ edn Vol. 1 (eds Marcus, R., Feldman, D. \& Kelsey, J.) 557-567 (Academic Press, San Diego, 2001).

11. O'Neill, T. W. et al. The prevalence of vertebral deformity in European men and women: the European Vertebral Osteoporosis Study. J. Bone Miner. Res. 11, 1010-1018 (1996).

12. Jones, G. et al. Prevalent vertebral deformities: relationship to bone mineral density and spinal osteophytosis in elderly men and women. Osteoporos. Int. 6, 233-239 (1996).

13. Cooper, C., Atkinson, E. J., O'Fallon, W. M. \& Melton, L. J. Incidence of clinically diagnosed vertebral fractures: a population-based study in Rochester, Minnesota, 1985-1989. J. Bone Miner. Res. 7, 221-227 (1992).

14. The European Prospective Osteoporosis Study (EPOS) Group. Incidence of vertebral fracture in Europe: results from the European Prospective Osteoporosis Study (EPOS). J. Bone Miner. Res. 17, 716-724 (2002).

15. Johnell, O., Gullberg, B., Allander, E. \& Kanis, J. A. The apparent incidence of hip fracture in Europe: a study of national register sources. MEDOS Study Group. Osteoporos. Int. 2, 298-302 (1992).

16. Elffors, I. et al. The variable incidence of hip fracture in southern Europe: the MEDOS Study. Osteoporos. Int. 4, 253-263 (1994).

17. Nagant de Deuxchaisnes, C. \& Devogelaer, J. P. Increase in the incidence of hip fractures and of the ratio of trochanteric to cervical hip fractures in Belgium. Calcif. Tissue Int. 42, 201-203 (1988).
18. Ross, P. D., Davis, J. W., Epstein, R. S. \& Wasnich, R. D. Pre-existing fractures and bone mass predict vertebral fracture incidence in women. Ann. Intern. Med. 114, 919-923 (1991).

19. Cuddihy, M. T., Gabriel, S. E., Crowson, C. S., O'Fallon, W. M. \& Melton, L. J. Forearm fractures as predictors of subsequent osteoporotic fractures. Osteoporos. Int. 9, 469-475 (1999).

20. Ismail, A. A. et al. Prevalent vertebral deformity predicts incident hip though not distal forearm fracture: results from the European Prospective Osteoporosis Study. Osteoporos. Int. 12, 85-90 (2001).

21. Lunt, M. et al. Characteristics of a prevalent vertebral deformity predict subsequent vertebra fracture: results from the European Prospective Osteoporosis Study (EPOS). Bone 33, 505-513 (2003).

22. Lindsay, R. et al. Risk of new vertebral fracture in the year following a fracture. JAMA $\mathbf{2 8 5}$, 320-323 (2001).

23. van Helden, S., Geusens, P. P., Winkens, B. \& Dinant, G. J. Clinical subsequent fractures cluster in time after first fractures. Ann. Rheum. Dis. 68, 99-102 (2009).

24. Center, J. R., Bliuc, D., Nguyen, T. V. \& Eisman, J. A. Risk of subsequent fracture after low-trauma fracture in men and women. JAMA 297, 387-394 (2007).

25. Johnell, O. \& Kanis, J. A. An estimate of the worldwide prevalence, mortality and disability associated with hip fracture. Osteoporos. Int. 15 , 897-902 (2004). 
26. Johnell, O. \& Kanis, J. A. An estimate of the worldwide prevalence and disability associated with osteoporotic fractures. Osteoporos. Int. 17, 1726-1733 (2006).

27. Cooper, C., Atkinson, E. J., Jacobsen, S. J., O'Fallon, W. M. \& Melton, L. J. Population-based study of survival after osteoporotic fractures. Am. J. Epidemiol. 137, 1001-1005 (1993).

28. US Department of Commerce. Hip fracture outcomes in people aged fifty and over: mortality, service use, expenditures, and long-term functional impairment (Office of Technology Assessment, Congress of the United States, Washington, DC, 1993).

29. Bliuc, D. et al. Mortality risk associated with lowtrauma osteoporotic fracture and subsequent fracture in men and women. JAMA 310 , 513-521 (2009).

30. Sernbo, I. \& Johnell, O. Consequences of a hip fracture: a prospective study over 1 year. Ostoeporos. Int. 3, 148-153 (1993).

31. Cameron, I. D. et al. Hip fracture causes excess mortality due to cardiovascular and infectious disease in institutionalized older people: a prospective five-year study. J. Bone Miner. Res. doi:10.1359/jbmr.091029.

32. Batsis, J. A. et al. Body mass index (BMI) and risk of noncardiac postoperative medical complications in elderly hip fracture patients: a population-based study. J. Hosp. Med. 4 E1-E9 (2009).

33. Hershkovitz, A., Polatov, I., Beloosesky, Y. \& Brill, S. Factors affecting mortality of frail hipfractured elderly patients. Arch. Gerontol. Geriatr. doi:10.1016/j.archger.2009.09.003.
34. Brauer, C. A., Coca-Perraillon, M., Cutler, D. M. \& Rosen, A. B. Incidence and mortality of hip fractures in the United States. JAMA 302 , 1573-1579 (2009).

35. Chrischilles, E. A., Butler, C. D., Davis, C. S. \& Wallace, R. B. A model of lifetime osteoporosis impact. Arch. Intern. Med. 151, 2026-2032 (1991)

36. Cooper, C. \& Melton, L. J. Vertebral fractures. BMJ 304, 793-794 (1992).

37. Harvey, N., Dennison, E. \& Cooper, C. in Primer on the Metabolic Bone Diseases and Disorders of Mineral Metabolism $7^{\text {th }}$ edn (ed. Rosen, C.) 198-203 (ASMBR, Washington, D.C. 2009).

38. Oleksik, A. et al. Health-related quality of life in postmenopausal women with low BMD with or without prevalent vertebral fractures. J. Bone Miner. Res. 15, 1384-1392 (2000).

39. O'Neill, T. W. et al. Back pain, disability, and radiographic vertebral fracture in European women: a prospective study. Osteoporos. Int. 15, 760-765 (2004).

40. Cooper, C. Secular trends in osteoporotic fracture: myth or reality? Osteoporos. Int. 20 (Suppl. 1), S1-S2 (2009).

41. Cooper, C., Campion, G. \& Melton, L. J. Hip fractures in the elderly: a world-wide projection. Osteoporos. Int. 2, 285-289 (1992).

42. [No authors listed] Fractured neck of femur. Prevention and management. Summary and recommendations of a report of the Royal College of Physicians. J. R. Coll. Physicians Lond. 23, 8-12 (1989).
43. Chevalley, T. et al. Incidence of hip fracture over 10-year period (1991-2000): reversal of a secular trend. Bone 40, 1284-1289 (2007).

44. Kannus, P. et al. Nationwide decline in incidence of hip fracture. J. Bone Miner. Res. 21 1836-1838 (2006).

45. Johnell, $\mathrm{O}$. The socioeconomic burden of fractures: today and in the 21st century. Am. J. Med. 103 (Suppl. 2A), S25-S26 (1997).

46. Burge, R. et al. Incidence and economic burden of osteoporosis-related fractures in the United States, 2005-2025. J. Bone Miner. Res. 22 , 465-475 (2007).

47. Cooper, C. Epidemiology of osteoporosis. Osteoporos. Int. 9 (Suppl. 2), S2-S8 (1999).

48. FRAX ${ }^{\circledast H O}$ Fracture Risk Assessment Tool [online] http://www.shef.ac.uk/FRAX (2009).

49. Kanis, J. A., Johnell, O., Oden, A., Johansson, H. \& McCloskey, E. FRAX and the assessment of fracture probability in men and women from the U. K. Osteoporos. Int. 19, 385-397 (2008).

50. McLellan, A. R., Gallacher, S. J., Fraser, M. \& McQuillian, C. The fracture liaison service: success of a program for the evaluation and management of patients with osteoporotic fracture. Osteoporos. Int. 14, 1028-1034 (2003).

51. Kanis, J. A. et al. Case finding for the management of osteoporosis with FRAXassessment and intervention thresholds for the U. K. Osteoporos. Int. 19, 1395-1408 (2008).

52. Sandhu, S. K. et al. Prognosis of fracture: evaluation of predictive accuracy of the FRAX algorithm and Garvan nomogram. Osteoporos. Int. doi:10.1007/s00198-009-1026-1027. 


\section{REVIEWS}

\section{CORRECTION}

Osteoporosis impact on health and economics

Harvey, N., Dennison, E. \& Cooper, C.

Harvey, N. et al. Nat. Rev. Rheumatol. 6, 99-105 (2010); doi:10.1038/nrrheum.2009.260

In the Review article by Harvey et al. published in the February 2010 issue of Nature

Reviews Rheumatology, the labels for the key in Figure 3 were inverted. Expected survival after osteoporotic fracture should be depicted by the solid line and observed survival by the dotted line. 